\title{
Una panorámica de la Economía del Turismo en España
}

\author{
Eugeni Aguiló \\ Catedrático de Economía Aplicada \\ Universidad de las Islas Baleares
}

\section{RESUMEN}

Este artículo pretende ser una presentación de este número especial de Cuadernos de Economía dedicado a la Economía del Turismo. Precisamente en su último epígrafe se hace una breve descripción del contenido de los seis artículos incluidos en dicho número. Además, se ofrece una panorámica de las publicaciones en revistas internacionales de economistas españoles desde el año 2000 al 2010. El indiscutible crecimiento en la década que finaliza de la investigación en esta área merece el tratamiento especial que se ha intentado ofrecer en el presente artículo.

Palabras clave: Economía del Turismo. Análisis Bibliográfico. España Clasificación JEL: L83

\section{INTRODUCCIÓN}

En el año 1997 Ginés de Rus y Carmelo León publicaban en la Revista de Economía Aplicada una muy meritoria panorámica de lo que era en aquel momento el estado actual de la Economía del Turismo, analizando lo que denominaron las áreas de investigación más consolidadas en el campo de esta disciplina. En la última parte del artículo exponían los trabajos más destacables realizados en España. Las aportaciones que atraían mayor interés por parte de los investigadores se inclinaban hacia el análisis del conocimiento de la demanda y a los impactos del turismo sobre 
la renta y el empleo de nuestra economía. Coincidiendo con la publicación de este artículo, el último tercio de la década de los noventa significó el punto de inflexión que marcaba el gran despegue de la investigación turística española reflejada en las publicaciones en revistas internacionales que con frecuencia han tenido en aquel momento o con el paso de los años índices de impacto.

Las razones que explican este cambio en España no resultan fáciles de entrever. En Aguiló (2008) se hacían ciertas cábalas sobre el hecho de que durante ésta década y, en especial, durante estos últimos años, los expertos económicos han otorgado al turismo incluso la capacidad de garantizar un comportamiento estable en la senda del crecimiento económico. En el pasado, el turismo era un sector importante en términos de PIB, pero hasta cierto punto un mal menor en su protagonismo en nuestro desarrollo económico. El final del largo periodo expansivo y el inicio de una grave crisis financiera y económica han reforzado la idea que ya tenían algunos de que el turismo no es un sector al que deban achacarse niveles intensos de volatilidad, ya que, en definitiva, salvo grandes shocks externos y situaciones catastróficas es capaz de proporcionar un crecimiento sin grandes oscilaciones al depender en muchos casos de las situaciones distintas que pueden producirse en una diversidad de países emisores de origen y que pueden compensarse entre sí.

También hay que citar un cierto desprestigio en la comunidad científica, en especial la económica, de la especialización en turismo, algo que no ocurría en otras latitudes o, en su caso, algo que se había superado mucho antes. Lo cierto es que a medida que avanzaba la década de los noventa esta opinión negativa se difumina y expertos con una elevada formación en sus campos genéricos de especialización encuentran en el turismo un sector rico en experiencias e información primaria y secundaria, bien para la aplicación de modelos o bien para el análisis de nuevos modelos con frecuencia aplicables a este sector a través de un profundo conocimiento de sus propios mecanismos, que en términos generales difieren de los que corresponden a otros sectores de la actividad económica.

La pujanza de las contribuciones españolas a revistas internacionales de turismo durante esta primera década del siglo XXI y su elevada tasa de crecimiento, no deja lugar a dudas si analizamos la aportación de Albacete y otros (2009). Los autores seleccionan una muestra de 25 revistas internacionales sobre turismo en las que existe algún autor español. Hay que señalar que durante el periodo considerado, 1998-2009, casi el $80 \%$ de los artículos se publicaron, por este orden, en las revistas: Tourism Management, Tourism Economics, Annals of Tourism Research, International Journal of Contemporary Hospitality Management y Journal of Travel Research. 
La senda de crecimiento de las publicaciones muestra una expansión extremadamente significativa como se muestra en el Gráfico 1. En una aportación, citada anteriormente, Aguiló (2008) señala que para el año 2008 un 12\% del total de artículos publicados en Tourism Economics, Tourism Management y Annals of Tourism Research eran de autores españoles. Recordemos que estas tres revistas están indexadas y son en las que más publican nuestros investigadores

GRÁfICO 1.-Evolución de las contribuciones en revistas de turismo en lengua inglesa

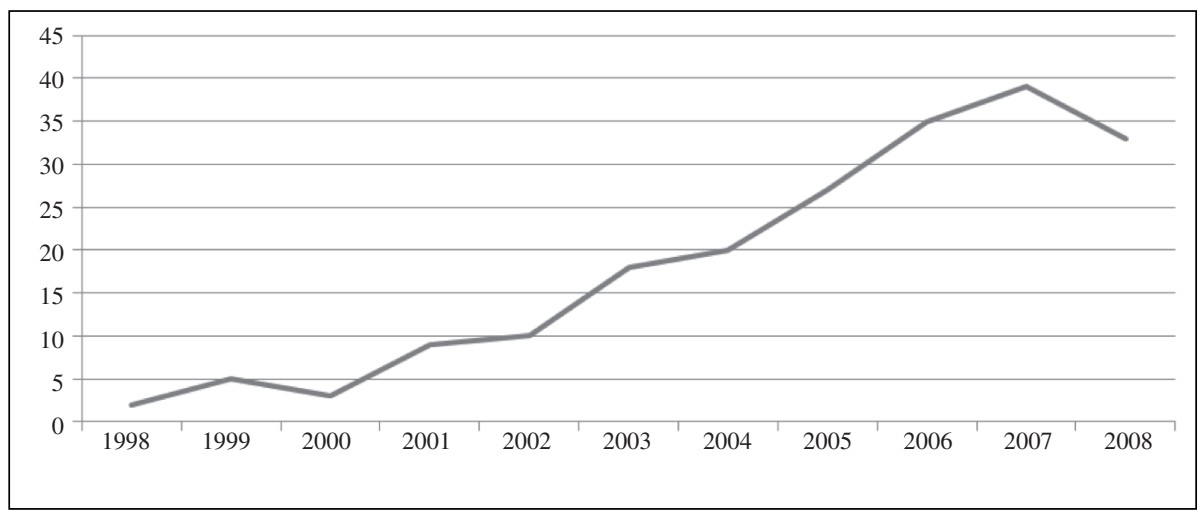

Fuente:.Albacete y otros (2009)

En este número especial de Cuadernos de Economía dedicado al turismo, es lógico que sólo se incluyan aportaciones al ámbito de la Economía del Turismo. Casualmente, además, en el caso español a diferencia de lo que puede ocurrir en otros países, la Economía del Turismo es la especialización que más destaca claramente sobre otras disciplinas turísticas en relación al número e importancia de las investigaciones publicadas. Únicamente las áreas de Marketing y, en menor medida, la Organización de Empresas alcanzan una cierta relevancia.

En el presente artículo haremos una síntesis de las aportaciones que se han desarrollado en el ámbito de la Economía del Turismo, y se han publicado básicamente desde el año 2000 por autores españoles, que como hemos señalado, supone el inicio de una década de un gran crecimiento en las investigaciones merecedoras de ser publicadas en dicho ámbito. Sólo haremos mención de aquellos artículos 
aparecidos en revistas internacionales de turismo y en algunas de carácter general también internacionales sin que ello quiera significar que en ciertos casos, aportaciones en revistas españolas hayan sido incluso merecedoras de publicación después de un proceso de revisión anónima. Es evidente que incluimos trabajos que en su gran mayoría han sido publicados por expertos pertenecientes a áreas de Economía Aplicada y Fundamentos del Análisis Económico incluyendo los que están trabajando en temas estrictamente vinculadas a la Estadística y la Econometría. Incluimos también algunos artículos publicados por expertos en Organización de Empresas, Marketing y otras áreas cuando su temática pertenezca a la economía o a temas tratados por especialistas de las áreas de economía anteriormente citadas.

Para desarrollar nuestro análisis incorporamos una división temática propia de la Economía del Turismo. No obstante, en algún caso incluimos algún área como los impactos económicos del turismo en la economía que son ineludibles aunque no existen muchas aportaciones españolas en revistas internacionales en el periodo analizado. Vamos en primer lugar a observar una seria de aspectos relativos a la demanda. A continuación, le corresponderá el turno a un conjunto dispar de temas que pueden agruparse en el entorno de la oferta. Un apartado especial merecerá el análisis de los mercados y precios Los impactos económicos del turismo se analizaron en cuarto lugar. A continuación, se hará una descripción de los temas medio ambientales turísticos y en último lugar se expondrá la panorámica del largo plazo relativa a la evolución de los destinos y el crecimiento que presentan elementos que son claramente fronterizos con el anterior. Existirá un apartado de otros para tratar aspectos no clasificables en estos apartados

\section{Demanda}

El análisis de la demanda es, sin duda, el área de investigación que ha sido objeto de mayor atención por parte de los economistas de nuestro país, si bien es cierto que en este caso existe coincidencia con la literatura de expertos internacionales. Ya en la década de los noventas y en el contexto de la escasez de publicaciones españolas mencionado, González y Moral (1995) y (1996) publicaron sendos artículos que han ganado en prestigio con posterioridad en la literatura internacional referida a la modelización de la demanda y los modelos de previsión. Las autoras analizaron la demanda de turismo internacional a España utilizando modelos de series temporales estructurales con componentes no observados espe- 
cificados de forma estocástica, como el estacional. Los precios son fundamentales para explicar la demanda así como el componente de tendencia ${ }^{1}$.

A partir de aquí han proliferado aplicaciones de los económetras a datos turísticos fundamentalmente en el ámbito de la demanda. Para analizar su comportamiento a través de modelos causales que explicitan variables económicas o de modelos de series temporales, presentamos en un primer epígrafe un conjunto de aportaciones que en diferentes ocasiones tratan de derivar propuestas relativas a las predicciones sobre variables turísticas. Por su importancia en la realidad de los destinos se ha distinguido en un segundo epígrafe que trata de la estacionalidad. A continuación, haremos referencia a las aportaciones incluidas en el motor de la demanda que son las motivaciones y lo que de una forma $\mathrm{u}$ otra incide en la toma de decisiones del turista más allá de las variables estrictamente económicas, para finalizar con el resultado del viaje en términos de gasto y niveles de satisfacción y sus consecuencias.

\subsection{Modelos de demanda univariantes y multivariantes}

Aunque los modelos utilizados y sus especificaciones han sido diferentes, en primer lugar nos referiremos a aquellos trabajos que han utilizado datos de panel combinando series temporales con datos de corte transversal para solventar problemas de multicolinealidad y aumentar los grados de libertad. Ledesma y otros (2001) calculan las elasticidades a corto y largo plazo del turismo de Tenerife, detectando la incidencia significativa de una variable poco utilizada como es la de los gastos promocionales. Garín y Pérez Amaral (2000) analizan la demanda de diecisiete países para visitar España y obtienen una elevada elasticidad renta y un valor reducido para tipos de cambio y precios. De la misma forma aunque incorporando un extenso análisis del turismo internacional que visita Madrid, Garín (2004) estima la demanda a esta ciudad de dieciséis países de origen, destacando la importancia de la renta de los turistas y el coste de vida diferencial entre origen y destino. Posteriormente, Garín (2006), (2007) y (2009) hacen una nueva explotación de datos de panel para otros destinos pero utilizando un modelo dinámico.

1 Ya desde el principio se observó el indudable interés de estos trabajos. De ahí que pronto surgieran comentarios sobre lo novedoso de su aplicación al turismo y también sobre sus limitaciones. En este sentido, véase Young and Pedregal (1997) y Garcia-Ferrer y Queralt (1997). 
Para ello, el procedimiento de estimación es un método generalizado de momentos teniendo en consideración los efectos no observados específicos del país. En el primer caso, se trata de la demanda internacional a las Islas Canarias y la conclusión es que la variable retardada es significativa lo que permite deducir la elevada lealtad e importancia del boca a boca junto con el hecho de que el turismo es un bien de lujo y que son relevantes los precios relativos y el transporte. El segundo artículo se dedica al turismo alemán a las comunidades autónomas españolas con resultados similares a los de Canarias en torno a la consideración de bien de lujo, precios relativos y transporte. Se resalta la importancia de la variable retardada de demanda sobre la demanda actual En el tercer caso referido a Galicia se estiman dos modelos: demanda de las Comunidades Autónomas y demanda de 24 países extranjeros. También como en el caso anterior ambas son sensibles a renta y precios. Finalmente, el artículo de Garín y Montero (2007) obtiene la demanda a Baleares de 14 países de origen y se resalta como en Canarias la significación de la lealtad de los países de origen y los precios relativos.

Continuando en el ámbito de los datos de panel Martínez Roget y Rodríguez González (2006) lo aplican al turismo rural en Galicia, detectando como en el caso anterior una elevada elasticidad-renta, sin olvidar, además de otras variables económicas, la relevancia de la reputación y las peculiaridades del establecimiento. Otra aplicación se debe a Gil-Pareja y otros (2007) quienes estiman el efecto del euro en los flujos turísticos entre países integrados en la moneda única, llegando a la conclusión de que el turismo ha aumentado en un 6,5\% por esta razón. Siguiendo con el interés sobre la relevancia en el turismo de una moneda común, Santana y otros (2010) observan que es un factor importante en la determinación de las llegadas de turistas. En este sentido, apuntan que tipos de cambio menos flexibles promueven el turismo.

Con el fin de dinamizar la modelización de la demanda, Rossello y otros (2005) y Aguiló y otros (2005) ponen en práctica un modelo de difusión en el que se supone que la información se transmite a los turistas potenciales a través de diferentes canales de información. Combinándolo con un modelo tradicional para datos de Baleares, se evidencia que los turistas emprenden un proceso de información previo a la elección de un destino concreto. Este trabajo fue útil para que en el segundo artículo, se intentase analizar para Baleares los efectos en los precios de los impuestos turísticos sobre el alojamiento en el bien entendido que, como hemos dicho, resulta básica la información sobre los servicios del destino.

Para el análisis de otros tratamientos de la demanda podemos citar a Rossello (2001) quien haciendo uso de datos mensuales de llegadas de turismo alemán y británico a Baleares pretende determinar los puntos de giro mediante indicadores avanzados. 
También con datos mensuales de turistas extranjeros a las cinco regiones españolas más turísticas, Bartolomé y otros (2009) aplican técnicas econométricas financieras así como las volatilidades de las series.

Una nueva vía de análisis y predicción se concreta en la introducción de algoritmos genéticos como los expuestos por Hernández-López (2004) con el fin de predecir la demanda en Tenerife. Considera que los modelos tradicionales no nos permiten conocer los vectores sociodemográficos que definirán a los turistas futuros, en cambio los algoritmos genéticos complementados con dichos modelos tradicionales, abren una vía de conocimiento del tipo específico de turistas que nos visitarán. Más adelante Hernández-López y Cáceres (2007) han utilizado un algoritmo genético diferente añadiendo una matriz de transición para predecir los turistas que visitarán una zona específica. Por su parte, Álvarez y otros (2009) mediante un modelo genético para llegadas mensuales de turistas británicos y germanos a Baleares, plantean sus ventajas relativas, comparan concretamente sus resultados con modelos más usuales de previsión como las medias móviles y el ARIMA y concluyen sobre su utilidad en este campo

También frente a los modelos clásicos existen algunas aportaciones que incorporan la técnica de las redes neuronales para la predicción de la demanda que, como señalan Palmer y otros (2006), no precisa del tipo de relación entre variables o del tipo de distribución de datos, en cambio puede ajustar funciones lineales o no lineales sin necesidad de conocer previamente la función correspondiente. Medeiros y otros (2008) consideran la llegada diaria de pasajeros a los tres aeropuertos de Baleares para obtener intervalos de predicción sensibles, lo que precisa modelizar correctamente sus varianzas. De cualquier forma Álvarez y Rossello (2010) estudian la demanda británica mensual a Baleares incorporando variables meteorológicas que según sus resultados pueden aumentar el poder predictivo cuando se utiliza una red neuronal artificial aunque tomando como criterio de ajuste el error porcentual absoluto promedio.

\subsection{Estacionalidad}

A pesar de la importancia que los agentes sociales y, en general, la opinión pública han otorgado al problema de la estacionalidad por sus indudables consecuencias económicas y sociales, pocos trabajos han sido publicados sobre esta cuestión por economistas del turismo. En todo caso, se ha puesto énfasis en los métodos de medición que sólo han aportado un número reducido de resultados que puedan ser objeto de propuestas para la gestión pública y privada. 
Cuñado y otros (2004), (2005), (2008a) y (2008b), Gil-Alaña (2005) y (2009) y GilAlaña y otros (2004) incorporan modelos de integración fraccionaria estacional a series temporales mensuales excepción hecha de Gil-Alaña y otros (2004) que los aplican a series temporales trimestrales del turismo español. En este contexto, es de interés la determinación de los niveles de integración y, por tanto, el grado de persistencia de las llegadas a los destinos. En Cuñado y otros (2008b) para datos de llegadas en USA se detecta un "break" estructural para el 11 de Septiembre determinado endógenamente por el modelo que se cumple para la mayoría de países de origen. En cambio, en GilAlaña (2009) existe una sustancial reducción en el grado de persistencia en el caso de los ciudadanos americanos que viajan a Europa después de los acontecimientos del 11 de Septiembre.

Por otra parte, en Fernández-Morales (2003) y Fernández-Morales y Mayorga (2008) aplican la descomposición del índice de Gini de la concentración estacional. En el primer caso se comparan tres destinos y se concluye que los dos destinos maduros (Málaga y Almería) mantienen un elevado valor del índice de Gini que perdura en el tiempo, mientras que el diversificado (Granada) posee un valor significativamente inferior, manteniéndose en una fase de disminución. En el segundo caso se hace para datos mensuales de la Costa del Sol, obteniendo la estacionalidad global y los efectos marginales por nacionalidades lo que permitiría aplicarles políticas turísticas diferenciales. Una aportación final al estudio de la estacionalidad la encontramos en Bartolomé y otros (2009), quienes estiman tres modelos de volatilidad condicional univariante para llegadas diarias de pasajeros a Baleares y Canarias, obteniéndose la persistencia a corto y largo plazo así como los efectos simétricos y asimétricos de shoks positivos y negativos de igual magnitud sobre la volatilidad.

\subsection{Motivaciones del turista. Análisis de decisiones}

En el análisis de la demanda, los expertos económicos han incidido también en el amplio abanico de motivaciones que conducen a las decisiones del turista que lógicamente están condicionadas por factores estrictamente económicos. En este apartado incorporamos el análisis de las decisiones que conducen a la elección de determinados segmentos de mercadeo específicos que en la literatura española se han centrado básicamente en el turismo rural. Un primer elemento básico en la toma de decisiones trata de explicitar cuál es el proceso que determina la elección final del turista. Para ello, Eugenio-Martín (2003) disecciona las cinco etapas de este proceso que irían desde una inicial, como es si viajar o no en un determinado 
momento del tiempo, hasta la última que sería la elección final del destino y el modo de desplazamiento. Nicolau y Mas (2005) y (2008) insisten en el proceso de toma de decisión multietápico. En el primer caso se trataría de hacer vacaciones, si ir al extranjero o no y finalmente, si ir a un destino o a varios. En el segundo caso, se observa que en el proceso multietápico el carácter litoral del viaje precede al carácter urbano en la elección del destino

Uno de los aspectos que ha tenido un mayor nivel de persistencia al menos en las dos últimas décadas es la reducción de la estancia media. En este sentido, Alegre y Pou (2006) destacan el poder explicativo, en un destino maduro como Baleares, del perfil sociodemográfico del turista y de las características de las vacaciones. Se afirma además que la duración de la estancia es sensible a los cambios de precios. Por su parte, Martínez-García y Raya (2008) observan en el caso del turista de low-cost, que las restricciones de tiempo son relevantes para explicar las diferencias en los días de estancia, así como la capacidad de gasto y el hecho de si el turista es urbano o de "sol y playa".

El estudio de las características sociodemográficas tiene su origen en nuestro caso en el artículo de Juaneda y Sastre (1999) que proporcionan en el ámbito de la segmentación importantes diferencias entre los turistas británicos y germanos que visitan Mallorca. Estas diferencias se observan en la elección de hoteles, hospitalidad, comida, etc. A partir de la Encuesta de Presupuestos Familiares española, Alegre y Pou (2004) analizan los factores que explican las decisiones de consumo de ocio y que serían el tiempo libre, factores culturales, edad, generación y la renta, aunque con una elasticidad menor que uno. Todo ello con un elevado nivel de heterogeneidad entre las familias. Con la misma, encuesta, Alegre y otros (2009), observando el significativo porcentaje de familias españolas que no gastan en servicios turísticos, analizan las determinantes económicas y sociodemográficos de la participación en las vacaciones y su intensidad que se mide por el número de trimestres que se gasta en este concepto. Variables sociodemográficos explicarían sólo la participación. Esta y su intensidad estarían condicionadas por la participación del año anterior y la renta, aunque de nuevo con una elasticidad menor que uno.

Mediando el condicionante de la restricción del presupuesto, Alegre y otros (2010) utilizan la muestra para España del Panel de Familias de la Comunidad Europea y, más específicamente, la pregunta sobre la capacidad de las familias para acceder a unas vacaciones anuales de una semana. La restricción mencionada no sólo está influida por la renta sino también por la capacidad de ahorro o el desempeño, aunque las variables como la edad, educación y salud determinan también la importancia con que las familias perciben su restricción presupuestaria. 
En cuanto a factores muy específicos que determinan o condicionan el viaje hay que citar a Nicolau y Mas (2006) y Nicolau (2008) y (2010). En el primer caso, se demuestra para una amplia muestra de turistas en España, que la influencia disuasoria de la distancia y los precios cuando se selecciona un destino es moderada por las motivaciones, en el sentido que éstas poseen un efecto disuasorio positivo o negativo. En el segundo caso y sobre la misma muestra, se detecta, una vez analizada la sensibilidad individual de la distancia, que esta influida por la renta, el número de hijos, el tamaño de la ciudad de residencia, el uso de intermediarios, las motivaciones etc. En el tercer caso, el autor concluye que la distancia no es disuasoria para toda la población, ya que, una vez más se modera por una serie de factores, como el comportamiento que se traduce en la búsqueda de la variedad de una vacación a otra. A su vez el comportamiento basado en la inercia refuerza el hecho de que la gente reduce sus preferencias por lugares distantes de sus destinos habituales y más próximos. Otro aspecto concreto en la decisión del turista fue estudiado por Araña y León (2008) cuando observaron en dos momentos distintos del tiempo la incidencia a corto plazo en destinos mediterráneos competidores y en las Islas Canaria de los sucesos del 11 de Septiembre. Concluyen que dicho fenómeno provocó un "shock" en la utilidad de los turistas y un cambio en el perfil de la imagen de los destinos, experimentando algunos un fuerte impacto negativo mientras otros respondieron positivamente.

Un aspecto de gran interés y que observa el fenómeno del crecimiento en la dirección opuesta, se encuentra en Eugenio-Martín y otros (2008) quienes analizan los requerimientos de desarrollo económico para atraer turismo, en tanto que éste puede influir en la toma de decisiones. Observan las diferencias entre países competidores en una gran área geográfica y concluyen la importancia del desarrollo económico sobre todo entre países en vías de desarrollo. Clavería y Datzira (2009), a través de un modelo de demanda agregada, describen la importancia de los factores macroeconómicos para determinar la demanda turística en Cataluña. Gil-Pareja y otros (2007) estiman el efecto positivo de las embajadas y consulados sobre los flujos turísticos en los países del G-7. Entre un $15 \%$ y un $30 \%$ dependiendo de la técnica de estimación, sería el valor de dicho efecto positivo. A su vez, el impacto es mayor para países en vías de desarrollo.

En cuanto a los elementos que motivan y condicionan la demanda de productos turísticos específicos es interesante observar en la literatura española el llamado turismo rural. Yagüe (2002) analiza los rasgos distintivos de lo que llamaba una nueva clase turística al referirse al turismo rural. Utilizando una encuesta a las poblaciones del Alto Palencia en la Región Valenciana, determina el perfil de dicho turista y sus características distintivas en relación al turismo tradicional. Destaca el hecho de que la renta y el nivel educativo no permiten diferenciar a estos dos tipos de turismo. Albaladejo y Díaz 
(2005) y (2009) mediante una encuesta a turistas en sus alojamientos de turismo rural en el Noreste de la Región de Murcia, analizan su perfil según cada tipo de alojamiento al obtener variables que indican una mayor probabilidad de influir en la elección que cada turista realiza cuando decide por un tipo concreto de alojamiento. Por su parte, Molera y Albaladejo (2007) con el fin de obtener un conocimiento más adecuado de la demanda de turismo rural, detectan la posibilidad de segmentar a los turistas en cinco grupos. Cuatro están motivados por la naturaleza, el entorno y el ambiente sosegado, a sólo dos les atraen las posibles actividades a realizar en el lugar, y al grupo más reducido sólo les motiva el pasar el rato con los amigos.

Para terminar este epígrafe, la referencia que resta tiene que ver con el turismo enológico que ha sido desarrollado por Marzo y Pedraja (2009), quienes intentan determinar que factores podrían tener impacto en las intenciones de participar en actividades turísticas relacionadas con el vino por parte de turistas potenciales que se sentían motivados por distintas actividades, sin que ni el coste, ni la distancia, ni el tiempo actuarían como barreras a su desarrollo.

\subsection{Gasto Turístico}

Los resultados de la actividad turística se deben considerar desde el punto de vista del destino y del propio turista. Como se ha repetido insistentemente, el énfasis en el volumen de turistas y de estancias debe ser modificado en el sentido de dar importancia al gasto como variable que nos dará la primera aportación a los beneficios para el destino.

En este ámbito hay que citar a Aguilo y Juaneda (2000) que utilizando modelos de regresión con variables ficticias relativas a las características de los turistas que visitan Baleares, obtienen significativas diferencias de gasto según distintos perfiles de turistas relacionados con dichas características (nacionalidad, edad, etc.). En cambio. Díaz-Pérez y otros (2005) detectan los segmentos de demanda en destino para determinar cuales llevan a cabo un mayor nivel de gasto en las Canarias. Particularmente interesante por la actualidad del tema de la discutida proliferación de "all-inclusive" en destinos maduros es el trabajo de Alegre y Pou (2008). Los autores con datos de Baleares concluyen que esta forma de alojarse supone una reducción de los ingresos del destino y un cambio en la distribución del gasto entre agentes económicos. Especialmente, el gasto en destino es claramente inferior. Para finalizar Palmer y otros (2006), utilizando así mismo datos de Baleares observan que los valores extremos del gasto medio por turista y día pueden invalidar el parámetro de localización de la media, aconsejando métodos 
alternativos para la estimación del parámetro. Se obtienen resultados con diferentes estimadores de localización, incluido el cálculo de sus intervalos de confianza.

\subsection{Niveles de satisfacción}

El objeto del viaje turístico estriba en obtener unos elevados niveles de satisfacción que condicionan una buena experiencia. En este sentido, Bigné y otros (2001), encuestando a turistas de una parte de la Costa Valenciana, mostraron la relación entre calidad y satisfacción y lealtad al destino y entre estas y otras variables del comportamiento del turista. La imagen es un antecedente de la calidad percibida, la satisfacción, la intención de volver y la recomendación. La satisfacción conduce a la disposición a recomendar. No se demuestra, en cambio, que la calidad influye en la disposición a recomendar y que la satisfacción provoque la intención a repetir el viaje. Por su parte, Campo y J B Garau (2008) muestran que al medir la satisfacción como un concepto multidimensional con datos de Baleares se pueden identificar las variables clave de la satisfacción total, obteniéndose diferencias en su generación dependiendo de las nacionalidades, lo que permitirá a los destinos mejorar sus políticas comerciales y de infraestructuras. Campos y Yagüe (2008) afirman que la supuesta relación negativa entre precio y satisfacción acaba siendo no significativa utilizando modelos lineales, ya que el efecto precio es doble sobre la satisfacción: positivo como indicador de calidad y negativo en términos de sacrificio.

En principio, puede postularse que la repetición y lealtad al destino se debe básicamente a la satisfacción experimentada. Alegre y Juaneda (2006), con datos de Baleares, muestran que la repetición, el conocimiento, y la calidad del destino pueden explicar los motivos del gasto turístico, en el sentido, de que los repetidores gastan menos que los visitantes por primera vez al poseer un conocimiento superior del lugar. La preferencia por la calidad y el sentido de apego al lugar suponen un gasto mayor. Alegre y Cladera (2006) utilizando también datos de Baleares muestran que a pesar de que un repetidor tiene una probabilidad mayor de repetir la visita, su principal determinante es un nivel elevado de satisfacción. Además de detectar que la repetición sólo tiene efectos limitados sobre la satisfacción, se indica la continuidad del interés por el sol y playa junto con la necesidad de ofrecer una calidad alta para mantener la lealtad. Finalmente, Alegre y J. Garau (2010) alegan que se debe reconsiderar la estructura tradicional de las encuestas de satisfacción, que sólo incluyen aspectos relativos a la satisfacción, obviando los de descontento que deberían ser incluidos explícitamente. En este sentido, demuestran que las situaciones negativas 
especialmente, el exceso de desarrollo o la congestión pueden ser decisivos para no repetir la estancia. El turista realiza una valoración dual compleja de las características del destino.

\section{OfERTA}

En el lado de la oferta la bibliografía económica del turismo no ha proliferado tanto como en el lado de la demanda y, además, se ha concentrado en una serie de temas específicos, entre los que destaca la formación del capital humano y, casualmente, en temas de género.

\subsection{Formación}

En términos muy generales, ha existido una realidad potencialmente contradictoria en el ámbito del sector turístico. Por un lado, muchos países y destinos turísticos han crecido en base a la existencia de unos recursos naturales y culturales aptos para su desarrollo, al mismo tiempo que se consideraba que ello podía absorber un volumen importante de mano de obra poco cualificada. Paradójicamente, todos los destinos y no sólo los maduros han venido insistiendo en la necesidad de formación turística para mejorar la productividad y la calidad en el servicio. Frente a este problema, los agentes han emprendido programas formativos a todos los niveles en los que abunda la formación en el ámbito de la propia empresa. En este contexto, una primera aportación se debió a Ramos y otros (2004) que a través de una base de datos originada en una encuesta de empresarios y trabajadores hoteleros de Baleares, obtienen características del empleo y diferencias en el capital humano según niveles de educación y formación en el puesto de trabajo por categorías de hoteles. El resultado es que los niveles educativos no impiden la movilidad entre categorías y que la formación en el lugar de trabajo juega un papel en las estrategias de desarrollo alternativas. Marchante y otros (2005) señalan que los desajustes educativos y otros componentes del capital humano en un número muy elevado de trabajadores en hoteles de Andalucía tienen impacto en los salarios de forma que la sobre-educación genera una prima positiva en el salario, aunque también otros tipos de educación como las lenguas, la educación profesional específica, etc. reciben recompensa en el mercado de trabajo. Por su parte, Lillo y Ramón (2005) incluyen diferentes variables para explicar los salarios de la educación y la experiencia, concluyendo que, en el 
caso español, el sector valora menos la educación que otros sectores servicios, que el tamaño de la empresa juega un importante papel y que los ingresos a lo largo de la vida son distintos según niveles educativos. Ahondando en este mismo objetivo, Lillo y Casado (2010) observan el efecto marginal de un año más de estudios en el incremento salarial en España y a diferencia de los resultados hasta aquí citados, se afirma que el sector turístico español no es diferente a la media de toda la economía. Sí es cierto que se produce una heterogeneidad cuando se desagrega el factor trabajo en edad, sexo y experiencia laboral.

Tratando un aspecto totalmente diferente, Cervera y Ruiz-Molina (2008) observan las potencialidades y las áreas de mejora del Grado de Turismo en el contexto del Espacio Europeo de Educación Superior, y señalan la necesidad de aproximar las diferencias entre académicos y profesionales en el diseño de los planes de estudios.

\subsection{Discriminación y Género}

Es interesante destacar la importancia relativa que ha tenido este problema en la literatura española. Incluso uno de los primeros artículos en revistas internacionales de turismo se debió a García-Ramón y otras (1995) quienes observaron en los casos de Cataluña y Galicia que el agroturismo sería una alternativa para la mujer en el intento de combinar responsabilidades domésticas con un empleo remunerado que permitiera mantener las pequeñas explotaciones y la conservación del entorno rural.

Ateniéndonos al periodo que nos ocupa en relación a las desigualdades por motivos de género, Tugores (2008), analiza para las Islas Baleares las diferencias en el sector turístico entre hombre y mujeres en horas trabajadas y remuneradas y el trabajo no remunerado en términos de responsabilidad familiar, lo que permite plantear los factores que pueden cambiar la situación actual. Muñoz-Bullon (2009) muestra que en el caso español las mujeres ganan un 6,7\% menos que los hombres, siendo las variables más explicativas el tipo de contrato, la cualificación requerida para el empleo y el subsector específico del que se trate. El hecho de que las diferencias salariales en turismo por diferencias no observables sea muy inferior que en el resto de industrias, puede explicarse por el hecho de que en un sector de bajos salarios como el turismo la legislación de salarios mínimos protege de hecho a la mujer. Campos-Soria y otros (2009) para una amplia muestra de hoteles y restaurantes en Andalucía, muestran que la segregación industrial y vertical, y, en menor medida, la de establecimiento aumentan las diferencias salariales. Aunque la horizontal y la segregación por categorías tienden a disminuirlas. El tratamiento de las condiciones 
de empleo y salarios en los hoteles y restaurantes españoles, partiendo del supuesto de que es un sector con alto empleo femenino y no cualificado, ha sido analizado por Fernández y otros (2010). De este supuesto se pueden deducir menores salarios y discriminación de genero, si bien es cierto que la incidencia de salarios bajos es menor en las regiones turísticas, aunque la discriminación de genero no depende de si la región es turística o no.

\subsection{Productividad y eficiencia}

El turismo como parte del sector servicios plantea dificultades a la hora de ofrecer un indicador de productividad, a ello se le une el hecho de que el producto turístico está compuesto por una multiplicidad de productos sensiblemente distintos. Such y Zamora (2006) utilizando el indicador de productividad aparente de los establecimientos de alojamiento, comparan lo ocurrido en las Comunidades Autónomas durante un periodo de más de cuarenta años, entre la productividad y el empleo.

Por otro lado, la siempre presente mirada hacia el problema de la productividad, ha motivado el interés por el grado de utilización de la capacidad y cómo le afecta a esta variable fundamental. De este modo, Roget y Rodríguez González (2006) descomponen el crecimiento de la productividad total del factor en progreso técnico, cambios de la eficiencia en la asignación, cambios en los niveles de ocupación, cambios en la eficiencia técnica y efectos del tamaño. Deducen que en el caso del alojamiento rural en Galicia la ocupación es el factor que más contribuye a la productividad total del factor. Pérez Rodríguez y Acosta (2007) analizan el coste de eficiencia y las economías

de escala en el sector de alojamiento de Gran Canaria. Observan economías de escala, excepto en el caso de empresas pequeñas y detectan que la ineficiencia de costes cambia en el tiempo, que no todas las empresas producen a costes mínimos y que dicha ineficiencia disminuye en el tiempo

\subsection{Calidad}

Al tratar la influencia de la calidad en las distintas variables que conforman los resultados de la empresa, se ha utilizado el término calidad de una forma genérica no sólo aplicada a la oferta sino también a la demanda o han recurrido a alguna variable concreta para medir la calidad. A nivel teórico, García y Tugores (2006), hacen uso de un modelo de duopolio en el que los hoteles compiten en calidad y precio con la 
inclusión de diferentes costes externos. Concluyen que soluciones eficientes incluyen la coexistencia de segmentos de alta y baja calidad y que una expansión del primero no supone necesariamente una mejora del bienestar. Prieto y González-Díaz (2008) se plantean si la calidad genera una renta económica en los hoteles insulares de Baleares y Canarias, afirmando que parece existir una relación entre ambas, si bien lo evidente es que la renta esta relacionada con la marca del hotel, ya que ésta última puede reducir los problemas de información asimétrica del turista al ofrecerles señales sobre estándares de calidad o disminuyendo la varianza en la calidad de un hotel al conocer ex ante sus características. Campos-Soria y otros (2005) distinguen entre efectos internos y externos para cuantificar la relación entre calidad y servicio y competitividad de los hoteles utilizando una encuesta a turistas alojados en hoteles de Andalucía. Los efectos internos se estimaron utilizando los costes medios directos y los externos por los efectos de la satisfacción en las ventas y la disposición al pago del cliente. Una calidad del servicio elevada influye significativamente en la competitividad. Finalmente, y aún tratándose de un trabajo sobre estacionalidad desde el lado de la oferta, Capó y otros (2007) con datos de Baleares, demuestran que hoteles con mayor nivel de servicios medidos en términos de categoría del alojamiento, abren un periodo mayor de tiempo durante el año.

\subsection{Innovación}

Otro factor de competitividad es la innovación, que por el interés que ha despertado, relatamos de forma separada. Pese a los problemas que presenta el estudio de la innovación en el sector servicios y, por tanto, en el turismo del I+D+i, se ha demostrado que las empresas turísticas innovan por la necesidad de generar condiciones de mayor competitividad y, por ello, algunos investigadores han prestado atención al tema.

Si el hecho de que las empresas turísticas innovan, como se demuestra en un estudio piloto para Baleares de Jacob y otros (2003), puede incluso sorprender, aunque parece lógico que las innovaciones se den en los ámbitos de los procesos, la distribución y la organización. Las empresas de alojamiento suelen hacer innovaciones no tecnológicas, estando orientadas en el caso de las tecnologías a las tecnologías de la información y comunicación. De nuevo, Jacob y Groizard (2007) trasladan el ejercicio anterior a hoteles de República Dominicana y México para estudiar las diferencias con los hoteles de Baleares de las mismas cadenas. Los resultados son que el tamaño y la innovación están relacionados y que mayor experiencia tiende a generar más innovación. Los establecimientos latinoamericanos desarrollas más innovaciones de proceso y organizati- 
vas que las de Baleares e incluso aquellos colaboran en la introducción de innovaciones en empresas locales. Para otra muestra de hoteles en Baleares, Orfila-Sintes y otros (2005) concluyen que hoteles de más categoría, los que pertenecen a una cadena y que están con contrato de gestión producen más innovaciones. La industria hotelera, además, innova al introducir tecnología incorporada en lugar de desarrollar actividades internas de I+D.

Un artículo pionero en el análisis de las innovaciones referidas al medio ambiente es el de González y León (2001), ya que, como veremos más adelante, la gestión reguladora y voluntaria de la incorporación de medidas medioambientales en la empresa ocupa una parte significativa de la literatura. En este artículo, los autores demuestran que las medidas adoptadas por hoteles y apartamentos de Gran Canaria son las que suponen una menor inversión y una reducción de costes. Fijan un índice de calidad ambiental determinado por factores objetivos del establecimiento y por las percepciones de los gestores y los clientes

Finalmente, Prats y otros (2008) destacan el papel jugado por las innovaciones y las relaciones entre agentes en la evolución de los destinos, haciendo uso de los conceptos de sistemas de innovación y redes sociales aplicadas al turismo a través del Sistema de Innovación Local Turístico. Aplican el Sistema a la Costa Brava central para valorar su capacidad innovadora y diseñar las estructuras de relaciones en red que favorecen la innovación.

\section{MERCADOS Y PRECIOS}

A nivel general la temática de la existencia de poder monopolístico en los mercados turísticos ha perdido, hasta cierto punto el interés que despertaba en décadas pasadas. Parece evidente que la aparición a través de la red de nuevos canales de distribución ha ampliado el mercado relevante, de forma que en el gran duopolio europeo actual de tour operadores, su aparente poder de monopolio esté suavizado por la presión competitiva por el uso de crecientes de Internet.

La determinación de los precios de los paquetes y los hoteles y la influencia de factores relativos a ambos ha abierto el camino para la introducción del modelo de precios hedónicos. P.M. Aguiló y otros (2001) lo utilizan para describir la influencia de los servicios contratados y su diversidad de características para las paquetes de tour operadores alemanes en Mallorca. Es importante en estos estudios, como también el de Aguiló y otros (2003), el detectar las diferencias entre tour operadores en este caso también los británicos, con el fin de conocer si existe un poder de mercado olipolístico 
por parte de los de mayor tamaño. El trabajo de Espinet y otros (2003) aplica el modelo a hoteles de la Costa Brava para ver las significativas diferencias de precios por categorías y el efecto de características como el tamaño, distancia a la playa, etc. Alegre y Sard (2009) observan la existencia de dispersión de precios de paquetes británicos y alemanes en Baleares. Las diferencias en los precios medios de los tour operadores persisten en el tiempo, así como también en las distribuciones de precios con distintas dispersiones y formas para los tour operadores y los mercados.

La globalización y sus consecuencias en el reforzamiento de la competitividad turística obligan a las empresas a diseñar estrategias que intentan identificar el grado de interrelación entre mercados turísticos. En este sentido, Grande y otros (2009) estudian las relaciones dinámicas a corto y largo plazo entre destinos españoles y su grado de interdependencia, junto a las relaciones de liderazgo potencial entre ellos. Poater y Garriga (2009) obtienen información de precios y de calidad mediante un coeficiente por estrellas durante el fin de semana. La información se obtiene a través de Internet e incluye a veinte ciudades europeas. Los destinos de "sol y playa" ofrecen precios más baratos, si bien en las ciudades del sur europeo son similares o incluso a veces mayores que las ciudades nórdicas.

El análisis del funcionamiento del mercado de trabajo turístico tiene su expresión en el artículo de Marchante y otros (2006) que detallan a través de una amplia muestra de hoteles y restaurantes en Andalucía de los determinantes de la dificultad de acceder a puestos de trabajo desocupados y de la escasez de puestos especializados. El salario neto por hora es el instrumento para corregir estos problemas, aunque influyen también aspectos como las tasas de desempleo, el nivel de actividad de las empresas, su localización, etc.

\section{IMPACTOS ECONÓMICOS DEL TURISMO.}

Es cierto que el repaso sobre la literatura turística especializada muestra que los impactos del turismo sobre las variables económicas de renta, empleo, ingresos fiscales, etc., ha estado muy presente en la literatura. No obstante, si echamos una ojeada a los autores españoles vemos que la importancia que se le da en el ámbito de las publicaciones españolas, incluidas las que realizan instituciones como Exceltur en el caso de Impactur, no se ha traducido en un número considerable de publicaciones en revistas internacionales. Por ello, su revisión va a ser necesariamente breve. Un análisis de carácter general lo encontramos en el artículo de Libreros y otros (2006), al estudiar cómo se han aplicado las Cuentas Satélites del Turismo en 62 países, que han utilizado 
la metodología armonizada de la Organización Mundial del Turismo del año 2001. De cualquier forma, se detecta la existencia de variantes de la metodología estándar al tratar de interpretar determinadas variables que hacen difícil realizar comparaciones entre países.

A nivel de impactos regionales hay que señalar los trabajos de Clemente y Valle (2008) y Clemente y otros (2008) referidos a Baleares. El primer artículo utiliza las tablas input-output de Baleares, incorporando, además, supuestos alternativos sobre la endogeneidad de componentes de la demanda final. Estiman el efecto económico de un descenso del $10 \%$ en los flujos turísticos comparando los resultados con los que se hubieran obtenido mediante el modelo de la matriz de contabilidad social. En el segundo artículo, utilizando las mismas técnicas, se trata de discernir los efectos económicos sobre renta y empleo que tendría una potencial sustitución de hoteles de baja categoría por hoteles de cuatro y cinco estrellas, incorporando diferentes escenarios. También se incluyen análisis de un cambio en la distribución del gasto turístico sobre la oferta complementaria. Valle y Nelson Yobesia (2009) examinan el impacto del turismo en la producción, el valor añadido y el empleo en Kenia mediante la Matriz de Contabilidad Social, demostrando que los servicios y en especial el turismo generan una actividad económica importante con un amplio potencial futuro de desarrollo.

Valdes y otros (2007) plantean, en primer lugar, una estimación del gasto turístico en Asturias a través del gasto realizado en los establecimientos de alojamiento colectivo y utilizando un modelo basado en técnicas no paramétricas. A partir de aquí el modelo input-output permite conocer los efectos en las variables económicas más significativas. En este apartado, resta por comentar el trabajo de Murillo y otros (2008) que analizan el impacto del entorno monumental de la Alhambra y el Generalife en la renta y el empleo directos, indirectos e inducidos en el caso de la renta. En la metodología incorporan entre otros el modelo input-output, constituyendo un ejercicio del vigor del turismo para proporcionar importantes relaciones intersectoriales.

\section{VALORACIÓN Y GESTIÓN DEL MEDIO AMBIENTE}

La literatura española ha respondido al interés que ha despertado entre los investigadores del turismo en general sobre la temática medio ambiental. En este capítulo vamos a ceñirnos básicamente a aquellos problemas del medio ambiente local que requieren una evaluación como consecuencia no sólo del hecho de ser un recurso fundamental para la sostenibilidad, sino también porque su disponibilidad no responde al funcionamiento del mercado al fallar este a la hora de limitar su uso. Ello genera la 
necesidad de su gestión pública, pero no sólo pública, y en algunas circunstancias a su regulación. Determinados aspectos medioambientales serán incluidos en el último epígrafe relativo a la competitividad y el crecimiento.

\subsection{Valoración del medio ambiente}

Al desarrollar las experiencias turísticas en un tema tan relevante para la economía medioambiental hemos de empezar citando el trabajo de Riera (2000) que utiliza el método del coste del viaje para medir el valor que los turistas otorgan a los servicios recreativos de un conjunto de zonas protegidas de Mallorca, a través de la demanda de turistas que generan y los beneficios que a ellos les proporcionan. A través de otro método de valoración, como es el método de valoración contingente aplicado a la pesca de altura de Canarias, León y otros (2003) calculan el valor de uso y de preservación que emanan de dicho recurso pesquero, destacando el hecho de que el valor de preservación de la biomasa es muy elevado en relación al valor de uso para la pesca.

También, González-Gómez y otros (2001) intentan evaluar los beneficios de usuarios y no usuarios de la zona turística de las Islas Cíes gallegas. La valoración social se obtiene a través de un análisis coste-beneficio, concluyendo el alto rendimiento que se podría obtener por euro gastado en su conservación. En un aspecto muy específico relativo a la observación de las preferencias de los turistas de segunda residencia en relación al resto de residentes sobre la calidad del agua del mar en una zona de Mallorca, Torres y otros (2009) llegan a la conclusión que la disposición a pagar para mejorar la transparencia del agua disminuye de forma no lineal cuando el agua se deteriora, aunque no se observan diferencias entre residentes permanentes y turistas residenciales.

\subsection{Imposición medioambiental}

Es lógico que si nos atenemos al ámbito económico de esta panorámica internacional, los expertos en análisis económico se hayan ceñido básicamente a las políticas impositivas y hayan dejado a otras áreas las disquisiciones sobre distintas políticas que pueden aplicarse al turismo. Aunque en este ámbito los estudios publicados son en parte aplicados, Rey-Maquieira y otros (2009) plantean un modelo dinámico de equilibrio general de una economía especializada en turismo con el fin de observar la influencia de dos políticas turísticas que afecten a la especialización turística y a la corrección de externalidades utilizando dos instrumentos: los impuestos turísticos y la aplicación de 
estándares de calidad. Este es más eficiente que el primero, aunque el segundo provoca distorsiones y puede generar un problema de sobreacumulación de capital.

Si nos atenemos al análisis aplicado, Palmer y Riera (2003) analizaron los efectos de la "Ecotasa" de Baleares, de amplia repercusión por lo novedoso de su implantación en una economía turística. El artículo propone la internalización de las externalidades medioambientales mediante un impuesto, analizando, no obstante, de forma crítica la implementación práctica de dicha "Ecotasa". Palmer y otros (2007) siguen valorando también en el caso de Mallorca los problemas de movilidad y congestión del tráfico en temporada alta producto, en parte, por el uso de los coches de alquiler para proponer un impuesto de tipo fijo a dichos vehículos, que si bien internalizaría poco las externalidades ocasionadas dada la baja elasticidad, constituiría, por la misma razón, un fondo elevado al que acudir para financiar alternativas al transporte privado. Una discusión más general por el ámbito impositivo que se enmarca, se encuentra en Gago y otros (2009) quienes analizan si la difusión de la imposición turística debe hacerse a través de la imposición general o debe ser específica. Se sugiere que ambos producirán una internalización de externalidades y un aumento de los ingresos sin afectar a la economía, aunque los impuestos indirectos turísticos serían más equitativos, neutrales y de más fácil aplicación.

\subsection{Gestión de impactos medioambientales}

El análisis que los economistas desarrollan en relación a los impactos medioambientales tiene un campo de actuación, además de la ya analizada valoración monetaria, en el de la compatibilización entre la propia incidencia medioambiental del impacto y la rentabilidad de las empresas y los destinos. De cualquier forma, esta compatibilidad puede ser afrontada mediante la planificación pública, pero también puede alcanzarse mediante la acción voluntaria de las empresas que analizan la posibilidad de obtener beneficios a corto y a largo plazo.

En este sentido, Martín Rojo (2009) introduce el análisis de la compatibilidad de la gestión medioambiental y los resultados económicos en el seno de un puerto deportivo de Andalucía, que es un producto capaz de plantear aspectos críticos de gestión medioambiental con el fin de mejorar la competitividad del sector náutico, a la vez que se pretende un desarrollo sostenible. Ruzumova y otros (2009) sostienen que si la calidad ambiental requiere intervención pública se puede producir un aumento de costes y, finalmente una perdida de competitividad. En este contexto el artículo analiza la hipótesis de Porter cuya propuesta trataría de suavizar o eliminar dicho trade-off. 
Dejando a un lado la regulación y situandonos en el ámbito de la actuación voluntaria, García Rodríguez y Armas (2007) destacan que en el sector hotelero español se da una estrecha relación entre la responsabilidad social medioambiental y los resultados empresariales. Blanco y otros (2009 a) y (2009 b) dedican sus esfuerzos a la mención de la literatura sobre las consecuencias económicas de actuaciones medioambientales voluntarias de las empresas turísticas. Las evidencias empíricas respaldan el hecho de la inexistencia de una penalización por el hecho de ser "verde" y confirman que el "free-rider" medioambiental no es algo tan común. Una aportación más concreta es la de Ayuso (2007) quien ya explícitamente describe instrumentos voluntarios concretos aplicados por el sector hotelero: códigos de conducta, sistemas de gestión medioambientales, etc. Se demuestra que los "ecolabels" y los sistemas de gestión medioambiental son los que más se aplican y aunque suponen costes superiores y de mayor complejidad que otros instrumentos, son los que más ofrecen beneficios tangibles e intangibles.

\section{CReCimiento y CiClo DE VIDA TURÍstico}

Un último gran epígrafe de la bibliografía especializada tiene que ver con el análisis a largo plazo que implica la discusión sobre el crecimiento y la sostenibilidad de los destinos entendida en un sentido amplio y comprehensivo. Esta temática ha estado vinculada lógicamente con el concepto de ciclo vital de los destinos que, al margen de su coherencia, plantea el análisis del estancamiento como etapa final del ciclo del producto turístico y los argumentos para la posible superación de esta última fase.

\subsection{Crecimiento}

Las cuestiones relativas al crecimiento se han sustanciado en diferentes ocasiones a través de la modelización de alternativas turísticas en la economía del destino. De este modo, Lozano y otros (2008) establecen un modelo de crecimiento medioambiental de una economía especializada en turismo para delinear su senda de crecimiento. Concluyen que no hay contradicción con la hipótesis del ciclo vital, ya que el deterioro medio ambiental y la congestión de bienes públicos son los principales argumentos para el estancamiento, aunque la calidad de los servicios privados juega un papel destacado en la evolución del destino. 
Ya a nivel aplicado, Balaguer y Cantavella (2002) afirman que el crecimiento económico español a largo plazo ha sido sensible a la expansión del turismo internacional, siendo fundamental el hecho de la competitividad exterior como variable básica de crecimiento. Capo y otros (2007 a) utilizan el modelo de Solow, aplicándolo a Baleares y Canarias a través de una serie de datos de tres décadas. Afirman que el turismo ha sido una fuerza decisiva para un desarrollo económico claramente por encima de la media española, si bien la reducción de su productividad puede amenazar un crecimiento en el futuro. Con el ejemplo, también, de Baleares y Canarias los mismos autores, Capó y otros (2007b) consideran que ambas economías pueden estar bajo el signo de la "enfermedad holandesa" que pudieron padecer determinadas economías sujetas a un rápido cambio con el fin de encontrar nuevas utilizaciones para exportar sus recursos naturales. En este contexto, se hace el parangón con economías turísticas y el uso creciente de recursos naturales para su crecimiento cuyo futuro puede estar comprometido

En una modelización de dos economías, una grande y rica que produce bienes de capital comercializables, Álvarez-Abelo y Hernández-Martín (2009) concluyen que si el turismo es un bien de lujo, el crecimiento diferencial a favor de la región pequeña es significativamente mayor. La relación real de intercambio mejora mucho a favor de estas y la inversión aumenta. A nivel municipal, Rigall y Fluvia (2007) y Rigall (2008) sostienen que dado que el producto turístico esta integrado en parte por bienes públicos, una oferta correcta de estos contribuye al desarrollo sostenible. En el primer artículo, al desarrollo teórico se le suma el cálculo a través de precios hedónicos de los precios hoteleros según su mejor o peor localización, lo que permite establecer que se crea un círculo virtuoso entre más precios, más ingresos y más posibilidades de ofrecer bienes públicos.

\subsection{Ciclo de vida}

Aunque la discusión sobre el modelo del ciclo de vida de Butler ha provocado que los investigadores se hayan esforzado durante tres décadas en escudriñar aspectos de su consistencia y aplicabilidad, hoy se ha convertido en un concepto útil para aludir al estancamiento inexorable o no de destinos sobre todo maduros. A nivel teórico, León y otros (2007) consideran el papel de la degradación ambiental y la disminución del capital natural como determinantes del ciclo de vida y el bienestar económico. El artículo afirma que la evolución del ciclo de vida del producto converge a la solución estacionaria caracterizada por niveles de consumo y capital natural positivos. 
A nivel aplicado se nos ofrece la posibilidad de examinar tres casos tan paradigmáticos como son Baleares, Benidorm y Tenerife en las aportaciones de Aguiló y otros (2005), Claver y otros (2007) y Oreja y otros (2008) respectivamente. Para Baleares se discuten algunas opiniones sobre lo inexorable de la fase de declive, afirmándose que el turismo de sol y playa evoluciona pero esta lejos de cambiar en la senda de los estereotipos del "nuevo turismo", ya que en Baleares se da un proceso de reestructuración para ofrecer más calidad y para garantizar que el modelo es sostenible a largo plazo. También en el caso de Benidorm se pone énfasis en la persistencia del modelo a través del estudio empírico de su evolución y su situación competitiva, insistiendo en la necesidad de que los hoteles adopten estrategias para mejorar la competitividad del destino. En lo que se refiere a Tenerife, los autores parten del modelo del ciclo vital junto a un modelo teleológico para explicar el proceso hacia la sostenibilidad de un destino maduro, en el que se integran aspectos estratégicos del segundo modelo para superar las limitaciones del modelo del ciclo vital. Consideran dos estrategias para Tenerife, político-legales para regular la actividad turística y decisiones para revalorizar la oferta.

\section{Otros}

En este último epígrafe se recogen un conjunto de aportaciones cuya temática ha sido estudiada por especialistas de diferentes disciplinas incluidas la Economía. En este sentido, destaca el análisis de la percepción que tienen los residentes sobre el desarrollo turístico, es decir, su impacto social. Aguiló y Rossello (2005) mediante una amplia encuesta a cerca de mil residentes en Baleares, dividen la población en cinco grupos de opinión siguiendo el análisis cluster. Destaca que a pesar del éxito económico de este destino maduro, existe un significativo porcentaje de críticos con sus resultados influidos por la fragilidad insular. Con el mismo objetivo, Oviedo-García y otros (2008) sostienen la correlación positiva entre la población que ha obtenido beneficios del turismo y su percepción positiva del desarrollo turístico y su planificación en una pequeña comunidad de Andalucía. Sin embargo, Diedrich y García-Buades (2009) no se quedan en el análisis de las percepciones, dando un paso más, al intentar detectar si estas pueden ser un indicador del declive de un destino en el sentido del modelo del ciclo vital. Sus resultados confirman la validez de la propuesta comparando cinco comunidades costeras de Belice.

Otras aportaciones han optado por describir aspectos parciales de las actitudes percibidas, bien sea por el impacto ocasionado, bien sea por el tipo de turismo que lo provoca. En la primera opción, Bujosa y Rossello (2007) se interesan por el impacto medioambiental y su percepción por los residentes en relación a la densidad de plazas hoteleras en diferentes 
municipios de Baleares. La conclusión es que a mayor densidad hotelera menor tolerancia de los residentes. En la segunda opción Bartolomé y otros (2009) inciden en el desarrollo de un nuevo producto turístico como es el turismo deportivo. Los residentes en Baleares perciben su desarrollo positivamente, aunque con significativas diferencias entre islas no sólo por su impacto sino también por el tipo de turismo deportivo a desarrollar.

Otro tema poco abordado por los economistas es la planificación turística. A destacar el trabajo de Pardellas y Padín (2005) reflexionan sobre la nueva configuración de la Euroregión Galicia-Norte de Portugal, aportando un modelo con una elaboración sistémica de este destino común a través de una estructura formal de sus elementos con el fin de ser útiles a gestores y planificadores en la toma de decisiones. Un aspecto más parcial de la planificación estratégica es el tema que desarrollan Vila y otros (2010) y que tiene que ver con la propuesta de un modelo preliminar del cuadro de mando (scorecards) equilibrado para los destinos turísticos. Se estudian las variables de comportamiento tradicional de dicho cuadro y se analiza cómo puede ser modificado mediante los resultados de una encuesta a más de 1500 municipios españoles con orientación turística.

Si bien los intentos de desarrollar indicadores tienen ya una larga tradición, el turismo ha ido incorporando recientemente el interés por ellos para medir la sostenibilidad multidimensional o, en su concepción más limitada, la que se refiere al aspecto medioambiental. Así lo hacen Pulido y Sánchez Rivero (2009), ya que al comparar diferentas enfoques teóricos, los autores aplican un índice de sostenibilidad turística global al sistema español de indicadores turísticos medioambientales. Por su parte, uno de los objetivos del artículo de Urtasun y Gutiérrez (2006) es construir un indicador de bienestar en términos de calidad de vida en base a indicadores parciales en las 50 provincias españolas, cuyo resultado determina la existencia de fronteras de capacidad de carga o niveles máximos que los destinos turísticos están dispuestos a soportar en términos económicos, socio-culturales y medioambientales. Haciendo uso de un aspecto muy específico, Rendeiro y Ramírez (2010) estiman un indicador de huella ecológica en Lanzarote relacionado con el transporte por carretera, evaluar su impacto y describir el "trade-off" entre necesidades de movilidad y desarrollo turístico en esta isla.

Finalmente, para terminar con este epígrafe de temas diversos, hacemos mención de aportaciones que tienen que ver con estrategias de comportamiento empresarial y que, en algunas ocasiones, han sido tratadas por expertos en Economía. Dos ejemplos significativos son el de Ramón (2002) que explica las posibles causas de la expansión internacional de las cadenas en términos de la teoría de la agencia, costes de transacción etc. y las decisiones en torno a la elección del modo de entrada en otros países, ya sea la propiedad o la gestión. El segundo ejemplo sería del Brida y otros (2010) que estudian el endeudamiento de las principales empresas hoteleras españolas, determi- 
nando una organización jerárquica y obteniendo clusters dinámicos que corresponden a sus estrategias de producción y endeudamiento.

\section{EL CONTENIDO DEL PRESENTE NÚMERO ESPECIAL}

En el presente número especial de Cuadernos de Economía dedicado a la Economía del Turismo se han incluido, además de este artículo introductorio, seis trabajos realizados por expertos de diversas universidades españolas. Dos de ellos tratan algunos aspectos específicos de la demanda. El artículo de Artal, García Sánchez y Navarro Azorín incorpora el tratamiento de la estancia media en un destino emergente de sol y playa, como es la región de Murcia, que ha mostrado en muchas zonas turísticas un descenso tendencial a lo largo de las dos últimas décadas. Su valor en términos absolutos depende, en el caso expuesto, tanto de los perfiles individuales del turista como de las propias características del destino. Por su parte Tamara de la Mata determina los factores que afectan a la decisión de elección del destino elegido por los residentes en España, procediendo a comparar las Comunidades Autónomas en lo referente a la propensión a viajar fuera de la región de origen y en qué medida las diferencias se deben a cuestiones geográficas, culturales o de otro tipo de factores.

De primordial importancia para el sector turístico es el tratamiento de la intermediación, sobre todo en un momento que está sufriendo profundos cambios. Calveras y Orfila incorporan la óptica del análisis económico, para examinar el significativo papel que aún juegan los tour operadores en el ámbito de los problemas de incertidumbre y el papel que está jugando Internet para promover la disminución de los costes de búsqueda. Aunque ya hemos señalado en otra parte de éste articulo, pocas son las aportaciones en revistas internacionales en el ámbito de los impactos económicos, sin embargo parece oportuno incluirlas dado su interés. En este sentido, Cañadas pone énfasis en el crecimiento extraordinario de países que ya disponen de Cuentas Satélite del Turismo. El artículo ofrece un estudio de las necesarias extensiones que se deben realizar a dicha cuenta, como son su adaptación al ámbito regional, la redefinición de los agregados macro y los modelos de impacto y los problemas metodológicos y estadísticos que plantea el tratamiento y medición de las segundas residencias en las Cuestas Satélite. Por su parte, Valdés, Aza y Baños estudian la evolución del impacto del turismo en Asturias durante el periodo 1998-2008, aplicando el análisis input-output a la medición del gasto turístico para conocer los efectos directos, indirectos e inducidos y su desagregación sectorial. Incluyen una investigación sobre la relación entre las magnitudes turísticas y el ciclo económico. 
Aportando una visión más a largo plazo, Pulido y Sánchez Rivero discuten cómo la competitividad y el crecimiento son factores claves para debatir sobre el papel del turismo en el desarrollo económico. No todos los factores de competitividad turística, como los definió el Foro Económico Mundial, tienen la misma capacidad para generar crecimiento turístico, por tanto los países han de optar por los mejores, sin olvidar el hecho de que no todos los países aprovechan igual su capacidad competitiva.

\section{REFERENCIAS BIBLIOGRÁFICAS}

Aguiló, E. (2008), "Presentación” XIII Congreso anual de AECIT. Vitoria.

Albacete, C.A. y M. del M. Fuentes-SÁez (2009), "Difusión de la investigación española sobre turismo en revistas internacionales" XIV Congreso anual de AECIT. Gijón

De Rus, G. y C. León (1997), "Economía del turismo. Un panorama” Revista de Economía Aplicada V (15): 71-109.

García-Ferrer, A. y R.A. Queralt (1997), "A note on forecasting international tourism demand in Spain" International Journal of Forecasting 13(4): 539-549

García-RAmón, M.D., G. Canoves y N. Valdovinos (1995) "Farm tourism, gender and the environment in Spain" Annals of Tourism Research 22(2): 267-282

González, P. y Moral P. (1995), "An analysis of the international tourism demand in Spain" International Journal of Forecasting 11(2): 233-251

González, P. y Moral P. (1996), "Analysis of tourism trends in Spain” Annals of Tourism Research 23(4): 739-754.

Young, P. y D. Pedregal (1997), Comments on "An analysis of the international tourism demand in Spain” by P. González and P. Moral International Journal of Forecasting 13(4): 551-556

\section{BIBLIOGRAFIA DE ECONOMIA DEL TURISMO EN REVISTAS INTERNACIONALES}

\section{Demanda}

\subsection{Modelos de demanda univariantes y multivariantes}

Aguiló, E., A. Riera y J. Rosselló (2005) "The short-term price effect of a tourist tax through a dynamic demand model: The case of the Balearic Islands" Tourism Management 26(3): 359-365 
Álvarez, M. y J. Rosselló (2010) "Forecasting British tourist arrivals in the Balearic Islands using meteorological variables" Tourism Economics 16(1): 153-168.

Álvarez, M., J. Mateu y J. Rossello (2009) "Forecasting tourist arrivals to Balearic Islands using genetic programming" International Journal of Computational Economics and Econometrics 1(1): 64-75.

Bartolomé, A., M. McAleer, V. Ramos y J. Rey-Maquieira (2009) "A risk map of international tourist regions in Spain" Mathematics and Computers in Simulation 79(9): 2741-2758

GARÍN, T. (2004) "Madrid as a tourist destination: analysis and modelization of inbound tourism" International Journal of Tourism Research 6(4): 289-302.

- (2006) "Inbound international tourism to Canary Islands: a dynamic panel data model" Tourism Management 27(2): 281-291.

- (2007) "German tourism”, Tourism Management, 28, (1): 12-22.

- (2009) "Tourism in Galicia: domestic and foreign demand", Tourism Economics 15 (4): 753769.

- y L.F. Montero (2007) "Tourism in the Balearic Islands: A dynamic model for international demand using panel data” Tourism Management 28(5):1224-1235.

- y T. PÉrez Amaral (2000) "An econometric model for international tourism flows to Spain" Applied Economics Letters; 7 (8): 525-529.

Gil-Pareja, S., R. Llorca-Vivero y J.A. Martínez (2007) "The impact of embassies and consulates on tourism" Tourism Management 28(2): 355-360.

HernándeZ-LóPEZ, M. (2004) “Future tourists' characteristics and decisions: the use of genetic algorithms as a forecasting method" Tourism Economics, 10(3): 245-262.

- y J.J. CÁCEREs (2007) "Forecasting tourists' characteristics by a genetic algorithm with a transition matrix” Tourism Management, 28(1): 290-297.

Ledesma, F.J., M. Navarro-Ibáñez y J. V. Pérez-Rodríguez (2001) "Panel data and tourism: a case study of Tenerife" Tourism Economics: 7(1) 75-88.

Martínez Roget, R. y X. A. Rodríguez GonzÁlez (2006) "Rural tourism demand in Galicia, Spain” Tourism Economics 12(1): 21-31(11).

Medeiros, C., M. McAleer, D. Slottje, V. Ramos y J. Rey-Maquieira (2008) “An alternative approach to estimating demand: Neural network regression with conditional volatility for high frequency air passenger arrivals" Journal of Econometrics, 147(2): 372-383.

Palmer, A., J.J. Montaño y A. Sesé (2006) "Designing an artificial neural network for forecasting tourism time series" Tourism Management 27(5): 781-790.

Rosselló, J. (2001) "Forecasting turning points in international visitor arrivals in the Balearic Islands" Tourism Economics: 365-380.

Rosselló, J., E. Aguiló y A. Riera (2005) “Modeling Tourism Demand Dynamics” Journal of Travel Research 44(1): 111-116. 
Santana, M.; F. J. Ledesma y J.V. Pérez-Rodríguez (2010) "Exchange rate regimes and tourism" Tourism Economics 16 (1): 25-43.

\subsection{Estacionalidad}

Bartolomé, A.; M. McAleer; V. Ramos y J. Rey-Maquieira (2009) "Modelling air passenger arrivals in the Balearic and Canary Islands, Spain" Tourism Economics 15(3): 481-500.

Cuñado, J.; L. A. Gil Alaña y F. Pérez de Gracia (2004) "Seasonal Fractional Integration in the Spanish Tourism Quarterly Time series" Journal of Travel Research, 42 (4): 408-414.

- (2005)" The nature of seasonality in Spanish tourism time series" Tourism Economics 11(4): 483-499.

- (2008a) "Persistence in international monthly arrivals in the Canary Islands" Tourism Economics 14 (1): 123-130.

- (2008b) "Fractional integration and structural breaks: evidence from international monthly arrivals in the USA" Tourism Economics 14(1): 13-24.

Fernández-Morales, A. (2003) "Decomposing seasonal concentration” Annals of Tourism Research, 30 (4): 942-956.

- y M.C. Mayorga (2008) "Seasonal concentration of the hotel demand in Costa del Sol: A decomposition by nationalities)", Tourism Management 29 (5): 940-949.

Gil AlaÑA, L.A. (2005) "Modelling international monthly arrivals using seasonal univariate long-memory processes" Tourism Management 26(6): 867-878.

- (2009) "Americans travelling to Europe: A new perspective based on persistence" Tourism \& Hospitality Research 9(1): 3-8.,

Gil-Alaña, L.A.; J. CuÑado y F. PÉrez de Gracia (2004) "Modelling monthly Spanish tourism: a seasonal fractionally integrated approach", Tourism Economics, 10(1): 79-94.

\subsection{Motivaciones del turista. Análisis de decisiones}

Albaladejo, I.P. y M.T. Díaz-Delfa (2005) "Rural tourism demand by type of accommodation” Tourism Management 26(6): 951-959.

- (2009) "Tourist preferences for rural house stays: Evidence from discrete choice modelling in Spain”Tourism Management 30(6): 805-811.

Alegre, J y J. Garau (2010) “Tourist satisfaction and dissatisfaction", Annals of Tourism Research, 37(1): 52-73.

Alegre, J. y L. Pou (2004) "Micro-economic determinants of the probability of tourism consumption", Tourism Economics, 10(2): 125-144.

— (2006) "The length of stay in the demand for tourism" Tourism Management 27(6): 1343-1355. 
Alegre, J., S. Mateo y L. Pou (2009) "Participation in tourism consumption and the intensity of participation: an analysis of their socio-demographic and economic determinants" Tourism Economics 15(3): 531-546.

- (2010) "An analysis of households' appraisal of their budget constraints for potential participation in tourism" Tourism Management 31(1): 45-56.

ARAÑA, J.E. y C.J. LEÓN (2008) “The impact of terrorism on tourism demand” Annals of Tourism Research 35(2): 299-315.

Clavería, O. y J. Datzira (2009) “Tourism Demand in Catalonia: Detecting External Economic Factors" Tourismos: An International Multidisciplinary Journal of Tourism 4(1): 13-28.

Eugenio-Martín, J.L (2003) "Modelling determinants of tourism demand as a five-stage process: A discrete choice methodological approach" Tourism \& Hospitality Research 4 ( 4): 341-355.

Eugenio-Martín, J.L.; N. Martín-Morales y T.M. Sinclair (2008) "The role of economic development in tourism demand" Tourism Economics 14 (4): 673-690.

Gil-Pareja, S.; R. Llorca y J. A. Martínez-Serrano (2007) "The Effect of EMU on Tourism" Review of International Economics, 15(2): 302-312.

JuAneda, C. y F. SASTRE (1999) "Balearic Islands tourism: a case study in demographic segmentation” Tourism Management 20(4): 549-552.

Martínez-Garcia, E. y J.M. Raya (2008) "Length of stay for low-cost tourism” Tourism Management 29(6): 1064-1075.

Marzo M. y M. Pedraja (2009) "Wine tourism development from the perspective of the potential tourist in Spain” International Journal of Contemporary Hospitality Management 21(7): 816-835.

Molera L. y I.P. Albaladejo (2007) "Profiling segments of tourists in rural areas of SouthEastern Spain” Tourism Management 28, (3): 757-767.

Nicolau, J.L. (2008) "Characterizing Tourist Sensitivity to Distance" Journal of Travel Research 47(1): 43-52.

- (2010) "Variety-seeking and inertial behavior: the disutility of distance" Tourism Economics 16(1): 251-264.

- y F. J. MAs (2005) "Stochastic modelling: A Three-Stage Tourist Choice Process" Annals of Tourism Research 32(1): 49-69.

Nicolau, J. L. y F. J. Mas y F. J. Mas (2006) “The influence of distance and prices on the choice of tourist destinations: The moderating role of motivations", Tourism Management, 27(5): 982-996.

- (2008) "Sequential choice behavior: Going on vacation and type of destination" Tourism Management 29(5): 1023-1034.

YAGÜE, R.M. (2002) “Rural tourism in Spain” Annals of Tourism Research 29(4):1101-1110. 


\subsection{Gasto Turístico}

Aguiló, E. y C. JuAneda (2000) “Tourist expenditure for mass tourism markets” Annals of Tourism Research, 27(3):624-637.

Alegre, J. y L. Pou (2008) "Tourism expenditure and all-inclusive packages - the case of a mature Mediterranean destination” Tourism Economics 14(3): 645-655.

Díaz Pérez, F.M., M.Bethencourt y J.A. Álvarez González (2005) "The segmentation of canary island tourism markets by expenditure: implications for tourism policy" Tourism Management 26(6): 961-964.

Palmer, A.; M. Beltrán y P. Cortiñas (2006) "Robust estimators and bootstrap confidence intervals applied to tourism spending" Tourism Management, 27(1): 42-50.

\subsection{Niveles de satisfacción}

Alegre, J. y M. Cladera (2006) "Repeat Visitation in Mature Sun and Sand Holiday Destinations”, Journal of Travel Research 44(3): 288-297.

Alegre, J. y J. Garau (2010) « Tourist satisfaction and dissatisfaction » Annals of Tourism Research 37(1): 52-73.

Alegre, J. y C. Juaneda (2006) “Destination loyalty. Consumers' Economic Behavior” Annals of Tourism Research 33(3): 684-706.

Bigné, J.E., M.I. SÁNCHEz y J. SÁnchez, J. (2001) “Tourism image, evaluation variables and after purchase behaviour : inter-relationship" Tourism Management 22(6): 607-616.

CAmpo, S. y J B GARAu (2008) "The Influence of Nationality on the Generation of Tourist Satisfaction with a Destination" Tourism Analysis 13(1): 81-92.

CAMPo, S. y M.J. YAGÜE (2008) "Effects of price on tourist satisfaction" Tourism Economics 14(3): 657-661.

\section{OfERTA}

\subsection{Formación}

Cervera, A. y M.E. Ruíz-Molina (2008) “Tourism education: a strategic analysis model” Journal of Hospitality. Leisure, Sport \& Tourism Education 7(2):59-12.

LiLlo, A. y J. Casado (2010) "Rewards to education in the tourism sector: one step ahead" Tourism Economics 16(1): 11-23. 
Lillo, A. y A.B. RAMón (2005) "Returns on education in the Spanish tourism labour market" Tourism Economics 11(1):119-132.

Marchante, A. J.; B. Ortega y R. Pagán (2005) "Educational mismatch and wages in the hospitality sector" Tourism Economics 11(1):103-117.

Ramos, V.; J. Rey-Maquieira y M. Tugores (2004) "The role of training in changing an economy specialising in tourism" International Journal of Manpower 25(1):55-72.

\subsection{Discriminación y Género}

Campos; J.A.; B. Ortega y M.A. Ropero (2009) "Gender segregation and wage difference in the hospitality industry", Tourism Economics : 847-866.

Fernández, M; Y. Pena y X. Pereira (2010) "Labor Conditions in the Spanish Hotels and Restaurants Industry" Tourism Analysis 14:293-312.

MuÑoz-Bullon, F. (2009) "The gap between male and female pay in the Spanish tourism industry” Tourism Management, 30(5):638-649.

Tugores, M. (2008) "Reconciling work and family from a gender perspective: an application to the Balearic hotel industry" Tourism Economics 14(1):223-239.

\subsection{Productividad y eficiencia}

Pérez Rodríguez, J.V. y E. Acosta (2007) "Cost efficiency of the lodging industry in the tourist destination of Gran Canaria (Spain)”, Tourism Management, 28(4):993-1005.

Roget, F. M. y X. A. Rodríguez GonZÁlez (2006) "Occupancy level and productivity in rural tourism establishments: the case of Galicia, Spain", Tourism Economics, 12(2):279-289.

Such, M.J. y M.M. Zamora (2006) "Spanish productivity" Annals of Tourism Research 33(3):666-683.

\subsection{Calidad}

Campos, J.A., L. González García y M. A. Ropero (2005) "Service quality and competitiveness in the hospitality sector" Tourism Economics 11(1):85-102(18).

CAPó, J.; A. Riera y J. Rosselló (2007) "Accommodation determinants of seasonal patterns" Annals of Tourism Research 34(2):422-436.

García, D. y M. Tugores (2006) “Optimal choice of quality in hotel services” Annals of Tourism Research 33(2):456-469.

Prieto, J. y M. González-Díaz (2008) “Is there an economic rent for island hotels?” Tourism Economics 14(1): 131-154. 


\subsection{Innovación}

Fernández, M., Y. Pena y X. Pereira (2010) "Labor Conditions in the Spanish Hotels and Restaurants Industry” Tourism Analysis. 14: 293-312.

González, M. y C.J. LEón (2001) “The adoption of environmental innovations in the hotel industry of Gran Canaria” Tourism Economics 7(2): 177-190.

JACOB, M. y J.L. GROIZARD (2007) “Technology transfer and multinationals: The case of Balearic hotel chains investments in two developing economies" Tourism Management 28(4): 976992.

Jacob, M., J. Tintoré, E. Aguiló, A. Bravo y J. Mulet (2003) "Innovation in the tourism sector: results from a pilot study in the Balearic Islands" Tourism Economics 9(3):279-295.

Orfila, F., Crespí, R. y E. Martínez Garcia (2005) "Innovation activity in the hotel industry: Evidence from Balearic Islands" Tourism Management 26(6): 851-865.

Prats, Ll.; J. Guia y F.X. Molina (2008) "How tourism destinations evolve: the notion of Tourism Local Innovation System” Tourism and Hospitality Research 8(3): 178-191.

\section{MERCADOS Y PRECIOS}

Aguiló P.M., J. Alegre y A. Riera (2001) "Determinants of the price of German tourist packages on the island of Mallorca", Tourism Economics 7(1): 59-74.

Aguiló, E., J. Alegre y M. SARd (2003) "Examining the market structure of the German and UK tour operating industries through an analysis of package holiday prices" Tourism Economics 9(3): 255-278.

Alegre, J. y M. SARD (2009) "Price Dispersion: An Empirical Analysis in the Tour Operating Industry" Tourism Analysis 14: 255-265.

EsPinet J.M.; M. SÁEz; G. Coenders y M. Fluvià (2003) "Effect on prices of the attributes of holiday hotels: a hedonic prices approach" Tourism Economics, 9(2): 165-177(13).

Grande, F-A., A. Muñoz y M.J. Botella (2009) "Flows into tourist areas: an econometric approach" International Journal of Tourism Research 11(1): 1-15.

Marchante, J.; B. Ortega y R. Pagán (2006) “Determinants of skills shortages and hard-to-fill vacancies in the hospitality sector" Tourism Management 27(5): 791-802.

PoAter, A. y A. Garriga (2009) "Tourism in European cities: insights into the dynamics of weekend hotel accommodation" Tourism Economics 15(1): 41-86. 


\section{IMPACTOS ECONÓMICOS DEL TURISMO}

Libreros, M., A. Massieu y S. Meis (2006) "Progress in Tourism Satellite Account Implementation and Development" Journal of Travel Research 45(1):83-91.

Murillo, J.; J. Romaní y J. Suriñach (2008) “The impact of heritage tourism on an urban economy: the case of Granada and the Alhambra" Tourism Economics 14(6): 361-376.

Polo, C. y E. VAlle (2008) "An assessment of the impact of tourism in the Balearic Islands" Tourism Economics 14(3):615-630.

Polo, C., V. Ramos, J. Rey-Maquieira, M. Tugores y E. Valle, (2008) “The potential effects of a change in the distribution of tourism expenditure on employment" Tourism Economics 14 (4):709-725.

Valdes, L., E. Torres y J.S. Domínguez (2007) "A model to study the economic impact of collective accommodation in a region" Tourism Management 28(1):152-161.

VAlle, E. y N. Yobesia (2009) "Economic Contribution of Tourism in Kenya" Tourism Analysis 14: 401-414.

\section{VALORACIÓN Y GESTIÓN DEL MEDIOAMBIENTE}

\subsection{Valoración del medio ambiente}

González-Gómez, M, X.M. González-Martínez, P. Polomé y A. Prada-Blanco (2001) "Evaluating public management in a tourism natural area: an application to the Cíes Islands" Tourism Economics 7(3): 251-265.

León C.J., J.E. Araña y A. Melián (2003) "Tourist use and preservation benefits from biggame fishing in the Canary Islands" Tourism Economics 9(1): 53-65.

Riera, T. (2000) "Mass Tourism and the Demand for Protected Natural Areas: A Travel Cost Approach" Journal of Environmental Economics and Management 39(1): 97-116.

Torres, C.M., T. Riera y D. García (2009) "Are preferences for water quality different for second-home residents?" Tourism Economics, 15(3): 629-651.

\subsection{Imposición medioambiental}

Gago, A.: X. Labandeira; F. Picosa y M. Rodríguez (2009) "Specific and general taxation of tourism activities. Evidence from Spain” Tourism Management 30(3): 381-392.

Palmer, T., A. Riera y J. Rosselló (2007) “Taxing tourism: The case of rental cars in Mallorca” Tourism Management 28(1): 271-279. 
Palmer, T. y A. Riera (2003) "Tourism and environmental taxes. With special reference to the "Balearic ecotax" Tourism Management, 24(6): 665-674.

Rey-Maquieira, J. J. Lozano y C. Gómez (2009) "Quality standars and versus taxation in a dynamic environmental model of a tourism economy" Environmental Modelling \& Software 24(12):1483-1490.

\subsection{GESTIÓN DE IMPACTOS MEDIOAMBIENTALES}

Ayuso, S. (2007) "Comparing voluntary policy instruments for sustainable tourism: The experience of the Spanish hotel sector” Journal of Sustainable Tourism 15(2):144.159.

Blanco, E.; J. Rey-MaQuieira y J. LozAno (2009 a) "Economic incentives for tourism firms to undertake voluntary environmental management" Tourism Management 30(1): 112-122.

- (2009 b) "The economic impacts of voluntary environmental performance of firms: a critical review” Journal of Economic Surveys 2(3): 462-502.

García Rodríguez, F.J. y M. Armas (2007) "Relation between social-environmental responsibility and performance in hotel firms" International Journal of Hospitality Management 26(4): 824-839.

Martín Rojo, I. (2009) "Economic development versus environmental sustainability: The case of tourist marinas in Andalusia” European Journal of Tourism Research 2(2): 62-177.

Ruzumova, M., J. Lozano y J. Rey-Maquieira (2009) "Is Environmental Regulation Harmful for Competitiveness? The Applicability of the Porter Hypothesis to Tourism" Tourism Analysis, 14(3): 387-400

\section{CRecimiento y Ciclo de Vida turístico}

\subsection{Crecimiento}

Álvarez-Albelo, C. y R. Hernández-Martín (2009) "Specialization in luxury goods, productivity gaps and the rapid growth of small tourism countries" Tourism Economics 15(3): 567-589.

Balaguer, J. y M. Cantavella (2009) “Tourism as a long-run economic growth factor: the Spanish case" Applied Economics 34 (7): 877-884.

CAPó. J., A. Riera y J.Rosselló (2007 a) "Tourism and long-term growth: A Spanish Perspective" Annals of Tourism Research 34(3): 709-726.

- (2007b) "Dutch Disease in Tourism Economies:Evidence from the Balearics and the Canary Islands" Journal of Sustainable Tourism 15(6): 615-627. 
Lozano J., C. Gómez y J. Rey-Maquieira (2008) “The TALC hypothesis and economic growth theory" Tourism Economics 14(4 ): 727-749.

Rigall, R. "Sustainable development in tourism municipalities: The role of public goods" Tourism Management 29(5): 883-897.

Rigall, R. y M. Fluviá (2008) "Public goods in tourism municipalities: formal analysis, empirical evidence and implications for sustainable development" Tourism Economics 13(3): 361-378.

\subsection{Ciclo de vida del producto}

Águilo, E., J. Alegre y M. SARd (2005) "The persistence of the sun and sand tourism model" Tourism Management 26(2): 219-231.

Claver, E., J.F. Molina y J. Pereira (2007) “Competitiveness in mass tourism” Annals of Tourism Research 34(3): 727-745.

León, C, J.M. Hernández y M. GonzÁLEz (2007) "Economic welfare, the environment and the tourist product life cycle” Tourism Economics 13(4): 583-602.

Oreja, J.R., E. PARra y V. YANES (2008) "The sustainability of island destinations: Tourism area life cycle and teleological perspectives. The case of Tenerife" Tourism Management 29(1): 53-65.

\section{OTros}

Aguiló, E. y J. Rosselló (2005) "Host community perceptions a cluster analysis" Annals of Tourism Research 32(4): 925-941.

Bartolomé, A., V. Ramos y J. Rey-Maquieira (2009). "Residents Attitudes towards Diversification: Sports Tourism in the Balearics" Tourism Recreation Research 34(1): 55-65.

Brida, J.G., L. Parte, W.A. Risso y M.J. Such (2010) "The international hotel industry in Spain: Its hierarchical structure" Tourism Management 31(1): 57-73.

Bujosa, A. y J. Rosselló (2007) "Modelling environmental attitudes toward tourism" Tourism Management 28(3): 688-695.

Diedrich, A. y E. GARcía-BuAdEs (2009) "Local perceptions of tourism as indicators of destination decline" Tourism Management 30(4): 512-521.

Oviedo, M.A., M. Castellanos y D. Martín (2008) “Gaining residents' support for tourism and planning" International Journal of Tourism Research 10(2): 95-109.

Pardellas, X.X. y C. Padín (2005) "The Minho Region (Galicia and Northern Portugal): A Case of Tourism Destination Planning” Tourism Analysis 10(2): 97-108. 
Pulido, J.I. y M. SÁnchez Rivero (2009) "Measuring tourism sustainability: proposal for a composite index" Tourism Economics 15(2): 277-296.

RAMón, A. (2002) "Determining factors in entry choice for international expansion. The case of the Spanish hotel industry" Tourism Management 23(6): 597-607.

RendeIRo, R. y P.P. RAMírez (2010) "Ecological footprint analysis of road transport related to tourism activity: The case for Lanzarote Island" Tourism Management 31(1): 98-103.

UrTasun, A. y I. GutiérRez (2006) “Tourism agglomeration and its impact on social welfare: An empirical approach to the Spanish case" Tourism Management 27(5): 901-912.

VILA, M., G. Costa y X. Rovira (2010) "The creation and use of scorecards in tourism planning: A Spanish example" Tourism Management 31(2): 232-239. 